\title{
REPRODUÇÃO DA APRENDIZAGEM HUMANA EM UMA AUTOMAÇÃO RESIDENCIAL POR MEIO DE MACHINE LEARNING
}

Matheus Felipe Terra-matheusfterra@hotmail.com

NuPSE - Núcleo de Pesquisas em Sistemas de Energia

Instituto Federal de Educação, Ciência e Tecnologia de Goiás, Câmpus Itumbiara

Rua Geraldo Ribeiro da Silva, 678

75523-150 - Itumbiara - Goiás

Marcelo Escobar de Oliveira - marcelo.oliveira@ifg.edu.br

NuPSE - Núcleo de Pesquisas em Sistemas de Energia

Instituto Federal de Educação, Ciência e Tecnologia de Goiás, Câmpus Itumbiara

Rua Getúlio Vargas, 418

75523-170 - Itumbiara - Goiás

Resumo: $O$ indivíduo humano está em constante aprendizado. Este ser observa o meio a que está inserido, identifica ações, analisa suas consequências, e desta forma consegue abstrair o conhecimento e definir padrões sendo esta sua forma de aprendizagem. Com o intuito de trazer uma inovação às formas de aprendizagem, têm-se investido muito em inteligências artificiais em prol de obter-se metodologias computacionais que possam representar este conhecimento de forma virtual. Um destes métodos é conhecido como Machine Learning, permite atribuir à máquina, diversas formas de se estabelecer o aprendizado. A exemplo têm-se o agrupamento, que se refere a captar informações, analisá-las, agrupar e identificar tendências, maneira semelhante à dos seres humanos em obter conhecimento. Uma das aplicações possíveis a esta inteligência, corresponde à automação residencial. Neste trabalho apresenta-se uma inteligência autônoma que capta informações comportamentais do usuário da residência e estabelece padrões, para que seja possível prever suas ações e funcionar de forma automática, estabelecendo-se assim uma residência inteligente e autônoma.

Palavras-chave: Inteligência artificial. Machine Learning. Aprendizagem. Conhecimento. Automação Residencial. 


\section{INTRODUÇÃO}

Os seres vivos inteligentes permanecem em constantes aprendizados. Os seres humanos, por exemplo, desde o seu nascimento passam por processos contínuos de estabelecimento do conhecimento. Um bebê inicia um processo individual em que aprende a utilizar o maxilar em movimentos verticais para triturar alimentos cada vez mais consistentes, a começar normalmente por leite materno, papinha, frutas moles e desta forma intensificam-se cada vez mais suas habilidades. Uma criança é treinada por professores para aprender a identificar padrões linguísticos por meio da análise de símbolos ordenados, processo conhecido como leitura.

Os indivíduos humanos adultos por sua vez, utilizam o ápice de sua cognição em prol de aprender novas habilidades que são utilizadas em sua carreira profissional. Aprendem por exemplo, como redigir um relatório importante para sua empresa, como utilizar um software para cálculo de seus lucros, assim como possui a habilidade de replicar novas receitas em suas refeições.

A evolução tecnológica constante que estamos vivendo tem permitido a representação computacional dessas formas de aprendizagem humana de uma maneira cada vez mais eficiente e usual. Frequentemente têm-se observado uma crescente procura e investimentos em pesquisas no ramo de inteligência artificial que busca trazer ao computador, maneiras de aprender e estruturar conhecimento de forma que se assemelhe ao indivíduo ou à estruturação social com que são organizados.

Uma técnica de inteligência artificial muito utilizada para atribuir metodologias de aprendizagem computacional, corresponde ao Machine Learning. Machine Learning é definido por Silva (2017) como uma maneira de atribuir ao computador, capacidade de realizar tarefas para as quais ele não foi programado previamente.

Desta forma, é possível aplicar em inúmeras áreas, metodologias semelhantes à utilizada por nós seres humanos com o intuito de permitir à máquina aprender, seja de forma autônoma, ou supervisionada por um "professor". Algumas das áreas que são mais aplicadas, correspondem à carros autônomos, identificação de fraudes digitais, processamento de imagens, sistemas de segurança, jogos de computadores, assim como automações residenciais, sendo este último, um exemplo da aplicação apresentada neste trabalho.

\section{METODOLOGIA DE PESQUISA}

Para desenvolvimento do projeto foi realizado o levantamento bibliográfico acerca da inteligência artificial e machine learning, e quais os principais modelos podem ser utilizados para reprodução do aprendizado humano. É importante ainda, no que tange à pesquisa bibliográfica, citar o levantamento sobre a metodologia de aprendizado dos seres humanos e a forma com que se estabelece, afim de se tornar possível sua reprodução por meio da computação.

Por último, ainda acerca do levantamento bibliográfico, levou-se em consideração o funcionamento e melhor custo-benefício dos componentes que foram utilizados no protótipo da automação residencial. Sendo, portanto, a metodologia de pesquisa, classificada como aplicada e exploratória, uma vez que visa gerar conhecimentos para aplicações práticas dirigidos à solução de problemas específicos, além de proporcionar maior familiaridade com este problema. 
Outro aspecto relevante da pesquisa, refere-se ao âmbito experimental, uma vez que se analisou e desenvolveu estratégias capazes de atingir o objetivo de reprodução computacional da aprendizagem. Para tal, realizou-se a esquematização e montagem de um protótipo de automação residencial e posteriormente o desenvolvimento de um software para controle.

Este software corresponde à inteligência autônoma, uma vez que executa ações por conta própria, baseado nos padrões comportamentais do usuário, que são constantemente estudados.

\section{INTELIGÊNCIA ARTIFICIAL}

Uma das definições usuais para inteligência artificial (IA) é como ramo da ciência da computação que se encarrega da automação do comportamento inteligente, de acordo com Luger (2014). Há diversas linhas de estudos envolvendo a IA visando diferentes aplicações. A vertente conexionista baseia-se na lógica e no funcionamento da mente racional. Há um esforço para alcançar a inteligência estruturando a arquitetura do cérebro humano. $\mathrm{O}$ modelo natural de neurônios demonstra a habilidade do cérebro de se adaptar ao mundo em que está inserido, criando e modificando conexões entre os neurônios, aponta Luger (2014).

Outro modelo inteligente baseado na organização biológica traz relação ao processo de interação entre espécies que se adaptam ao seu ambiente. "Desta forma, aplicam os princípios da evolução biológica aos problemas de encontrar soluções para problemas difíceis." (LUGER, 2014, p.14). Este método não busca a solução à questões raciocinando logicamente a respeito, pelo contrário, ele cria populações de respostas competidoras e as evolui para soluções melhores em um processo que imita a evolução biológica. O sistema social representa outra metáfora para a inteligência, uma vez que apresenta um comportamento em conjunto que permite trabalhar com questões que seriam improváveis à apenas um integrante.

Esta metáfora no meio acadêmico, estabelece o conceito de Inteligência Artificial Distribuída (IAD), que os autores Alvares e Sichman (1997), explicitam como um ramo muito promissor de pesquisa da inteligência artificial, e que ainda é pouco explorada atualmente. Ela se diferencia do modelo clássico de IA, porque o estudo tradicional busca analisar o comportamento individual humano, cujo foco está na representação do conhecimento e métodos de inferência. Já o modelo da IAD, é baseada no comportamento social, e seu foco está nas ações e interações entre os agentes, apontam Alvares e Sichman (1997).

Outra área da inteligência artificial que vem crescendo diz respeito a Machine Learning que corresponde à aprendizagem de máquina. É uma técnica que permite ao computador aprender por si só, sem a necessidade de um instrutor guiar seu conhecimento.

\section{MACHINE LEARNING}

O Aprendizado de Máquina ou Machine Learning corresponde à um campo da inteligência artificial que estuda e desenvolve algoritmos que permitem o computador a aprender e resolver problemas aos quais não foi programado previamente, afirma Silva (2017). Russell, Norvig e Intelligence (1995, apud Silva, 2017, p.34) dividem os treinamentos que a máquina pode ser submetida, em três campos, que são classificadas de acordo com o modelo de feedback que recebe durante seu treinamento, os seguintes campos são:

- Aprendizado supervisionado: São registradas por um treinador "humano" entradas e saídas como exemplos para que a máquina possa traçar padrões e assim ser ensinada a mapear uma regra geral para situações porvir; 
- Aprendizado por Reforço: O computador interage com situações e o meio de forma dinâmica, e recebe um feedback em forma de pontuação, afim de avaliar e ajustas pesos para cada ação, determinando assim, quais ações são válidas para seu aprendizado;

- Aprendizado não supervisionado: O computador não recebe saídas mapeadas, como é o caso do aprendizado supervisionado. Ele recebe entradas diversas, e a partir delas, constrói padrões e agrupamentos, afim de aprender por si só.

O ramo do Aprendizado não supervisionado tem uma crescente expansão de sua utilização no ramo computacional, devido a autonomia que é atribuído à máquina, sem a necessidade de um professor que a atribua valores às suas escolhas.

\title{
4.1 Aprendizado não supervisionado
}

O autor Luger (2014) em seu livro, explicita que o aprendizado não supervisionado, elimina o papel do treinador/professor, desta forma, o próprio algoritmo de aprendizagem avalia seus conceitos.

\begin{abstract}
A ciência talvez seja o melhor exemplo de aprendizado não supervisionado em seres humanos. Os cientistas não têm o benefício de um professor. Em vez disso, eles propõem hipóteses para explicar observações; avaliam as suas hipóteses usando critérios como simplicidade, generalidade e elegância e testam hipóteses por meio de experimentos que eles mesmos concebem. (LUGER, 2014, p.358)
\end{abstract}

Desta forma, ressalta-se a importância do estudo da forma de aprendizagem humana para reprodução computacional, assim como estabelecer de forma mais consistente a estruturação do conhecimento. Lakoff (1987, apud Luger, 2014, p.359) aponta que a categorização é fundamental à cognição humana, afirma ainda que o conhecimento teórico de alto nível está atrelado à capacidade de organizar particularidades das experiências em taxonomias coerentes. Logo, a formação do conhecimento se dá pelo agrupamento de suas experiências por semelhanças, sendo correspondente à técnica não supervisionada. Esta formação de categorias ou agrupamentos é a base para uma teoria unificada da descoberta científica desenvolvida por Nordhausaen e Langley (1990, apud Luger, 2014, p.359).

Portanto, um ramo utilizado para o estabelecimento do aprendizado da máquina, é por análises de tendências, através do agrupamento dos parâmetros. O algoritmo de machine learning analisa as informações, as agrupa de acordo com parâmetros semelhantes, e assim consegue traçar padrões. Técnica equivalente com a utilizada pelo ser humano para reconhecimento e aprendizagem.

\subsection{Agrupamento conceitual}

O objetivo do agrupamento é organizar as informações em classes que satisfaçam algum padrão de qualidade, otimizando o máximo possível a similaridade entre objetos da mesma classe, apresenta Luger (2014).

Os métodos numéricos, baseados na abordagem da taxonomia numérica, é uma das mais antigas técnicas para o agrupamento. Esta representação considera uma coleção de características e atribui valores a elas. Em seguida é aplicada uma métrica de similaridade, tratando paridades de objetos. A semelhança entre estes dois objetos corresponde à distância euclidiana entre eles em relação ao valor atribuído pelas características. 
Esta abordagem conhecida como agrupamento aglomerante, forma categorias da seguinte maneira, como apresentado por Luger (2014):

1- Examinar todos os pares de objetos, selecionando o par com o maior grau de similaridade, tornando-o um agrupamento.

2- Definir as características do agrupamento como uma função, tal como a média, das características dos membros componentes e, então, substituir os objetos componentes por essa definição do agrupamento.

3- Repetir esse processo sobre a coleção de objetos até que todos eles tenham sido reduzidos a um único agrupamento.

4- Muitos algoritmos de aprendizado não supervisionado podem ser vistos como realizando estimativas de densidade de máxima verossimilhança, o que significa encontrar uma distribuição da qual os dados pudessem ser retirados mais provavelmente.

Como resultado deste algoritmo, o autor ainda ressalta que este método representa agrupamentos extensionalmente, ou seja, por enumeração dos objetos.

Tendo em vista o conceito de inteligência artificial e sua técnica de aprendizagem, é possível aplicar a metodologia não supervisionada, que se assemelha à forma de estruturação do conhecimento humano, para obter informações, realizar previsões baseadas em dados estatísticos, assim como abstrair padrões e conceitos, por meio de agrupamentos de parâmetros.

Para tal, desenvolveu-se um software computacional que obtém informações comportamentais do usuário de uma residência por meio de sensores dispostos nela. De posse destas informações, analisa-se os padrões de comportamento, abstrai-se o aprendizado e o sistema é capaz de realizar ações, por meio de atuadores dispostos na residência, de forma semelhante ao usuário, construindo assim uma automação residencial inteligente e autônoma, capaz de aprender após estudar o histórico de ações.

\section{PROTÓTIPO DA AUTOMAÇÃO RESIDENCIAL}

Para realizar a identificação de padrões pela inteligência, foi desenvolvido um protótipo de automação residencial. Para tal, aplicou-se dispositivos IoT(Internet das coisas), para comunicação com o software que foi desenvolvido. Utilizou-se, por exemplo, as placas microcontroladas Arduino Mega e Uno para acoplamento dos sensores que ficam dispostos na residência, permitindo assim a disponibilização de tais informações à inteligência do software, assim como permite o envio de comandos do programa, até determinados atuadores para execução de algumas tarefas.

Diversos sensores com funções diferentes foram utilizados, como para captação da temperatura e da umidade, captação da intensidade luminosa, sensor de temperatura da água do chuveiro, sensor não invasivo de corrente e também de tensão. Já os atuadores correspondem à um módulo dimmer que possibilita o controle da potência que é liberada ao chuveiro e à lâmpada, para controle respectivamente, da temperatura da água e da iluminação. Assim como um LED infravermelho que permite o controle de dispositivos como o ar condicionado e televisão que são comandadas por infravermelho.

Este protótipo é capaz de captar informações dos cômodos da residência, assim como do consumo energético no decorrer do dia. Os sensores citados anteriormente, dispostos estrategicamente, são capazes de realizar medições programadas de parâmetros como 
temperatura, umidade, intensidade luminosa incidente, temperatura da água que sai do chuveiro, corrente consumida pelas cargas da residência, assim como da tensão disponível nas tomadas.

De posse destas informações, a inteligência é capaz de registrar em um banco de dados as ações que são exercidas pelo usuário na residência para estudo do seu padrão comportamental, afim de ser capaz de antecipar as ações, funcionando de forma autônoma. Alguns atuadores são dispostos também de forma estratégica ao redor do ambiente, para que seja possível exercer influência sobre o local. Desta forma é possível o controle do ar condicionado, controle da televisão, alteração da temperatura da água que sai do chuveiro e configuração da intensidade luminosa emitida pela lâmpada.

\section{O SOFTWARE DA AUTOMAÇÃO}

O software utilizado para controle da automação, foi desenvolvido na linguagem de programação Python. Faz-se uso de bibliotecas que permitem a comunicação serial entre o computador que executa o software e a placa microcontrolada Arduino, que é conectada via USB.

Uma vez estabelecida tal comunicação, o programa realiza medições dos sensores acoplados ao Arduino e apresenta tais informações por meio de dashboard. Permite, ainda, o controle manual (exercido pelo usuário por meio da interface de controle do programa) e automático (foco deste trabalho) onde a inteligência é capaz de realizar ações autônomas na residência, aplicando o aprendizado baseado nas informações comportamentais captadas do usuário.

\section{ABSTRAÇÃO DO CONHECIMENTO DE FORMAARTIFICIAL}

O software que realiza o controle da automação corresponde à inteligência do sistema. A cada quinze segundos o programa realiza requisições ao banco de dados. Uma das requisições diz respeito aos dados de histórico de consumo e a outra requisição corresponde aos registros dos padrões de ação do usuário. O sistema recebe como resposta uma matriz contendo todos os registros de histórico da utilização pelo usuário. Essa matriz, possui como linha valores correspondentes aos seguintes parâmetros, conforme a figura 1.

Figura 1 - Esquema representativo da Matriz Recebida.

Matriz Recebida $=\left|\begin{array}{ccc}\text { Horário } & \text { Ação } & \text { Valor } \\ 2020-02-10 \text { 15:48:52, } & \text { Ligar-Ar, } & 1 \\ 2020-02-22 \text { 18:50:33, } & \text { Ligar-Ar, } & 1 \\ 2020-01-1922: 37: 45, & \text { Ligar-Ar, } & 1 \\ \cdot & \cdot & . \\ \cdot & \cdot & . \\ 2020-02-10 \text { 15:40:12, } & \text { Ligar-Ar, } & 1\end{array}\right|$

Fonte: Elaborado pelo autor. 
Essa matriz corresponde ao dado sem tratamento, contendo todas as ações desempenhadas, conforme é armazenado no banco de dados. A partir dele é feito uma análise de dados, filtragens e agrupamentos, para identificar tendências correspondentes ao padrão de funcionamento do dispositivo.

A primeira ordenação que os dados são submetidos classifica-os por ações, e posteriormente em ordem crescente cronologicamente. Em seguida, é então executado um algoritmo que determina o dia da semana de ocorrência da ação, mediante a data a qual foi realizada. Após determinado o dia de ocorrência, a data é refatorada para que se leve em consideração apenas o horário de cada registro, resultando então em uma matriz com linhas no formato que se segue.

Figura 2 - Esquema representativo da Matriz Refatorada.

Matriz Refatorada $=\left|\begin{array}{cccc}\text { Dia } & \text { Horário } & \text { Ação } & \text { Valor } \\ 0, & 15: 40: 12, & \text { Ligar-Ar, } & 1 \\ 0, & 15: 48: 52, & \text { Ligar-Ar, } & 1 \\ \cdot & \cdot & \cdot & \cdot \\ \cdot & \cdot & \cdot & \cdot \\ \cdot & \cdot & \cdot & \cdot \\ 5, & 18: 50: 33, & \text { Ligar-Ar, } & 1 \\ 6, & 22: 37: 45, & \text { Ligar-Ar, } & 1\end{array}\right|$

Fonte: Elaborado pelo autor.

Por conseguinte, tem-se a fase de agrupamento. Para esta etapa, são feitas séries de comparações entre os horários e dias das ações. Caso os horários da comparação estejam no mesmo intervalo de 15 minutos e corresponderem ao mesmo dia da semana, significa que fazem parte do mesmo agrupamento, logo, há o acréscimo dos valores para intensificar a recorrência deste agrupamento. Esse intervalo de 15 minutos foi estabelecido, considerando um range de tempo em que duas ações podem ser consideradas como ocorridas em intervalos de tempo parecidos. Caso haja discrepância entre os requisitos, ou seja, uma ação dista mais de 15 minutos da outra, ou caso tenham ocorridas em dias diferentes, há a criação de um novo agrupamento, significando que há a ocorrência desta atividade em um período temporal diferente, e o mesmo deve ser levado em consideração na nossa análise estatística posterior.

Após realizado o agrupamento dos registros, tem-se duas opções para realizar a análise estatística. Pode-se utilizar uma matriz tridimensional, para armazenar os agrupamentos, considerando o dia, horário e quantidade de ocorrência, para cada seleção de valores. Embora funcional, há um aumento da dificuldade por parte da linguagem de programação para se trabalhar com este tipo de matriz, havendo uma incompatibilidade para trabalho matemático com as bibliotecas que estavam sendo utilizadas pelo trabalho. Para resolução, optou-se pela criação de três vetores relacionais, o que significa que as posições de vetores distintos possuem relação entre si. 
A partir destes três vetores criados, há uma nova ordenação dos grupos para posicionar os horários de forma crescente, e assim obter-se uma relação ordenada entre o dia da semana da ação, horário, e quantidade de vezes que a ação foi executada.

A última análise a ser feita, é uma quantificação estatística, onde o mesmo procedimento é realizado para cada um dos dias. É separado os valores de cada dia da semana e em seguida, soma-se a quantidade de ocorrências de cada um dos grupos, resultando em um total diário das ações. Divide-se então os valores de recorrência dos agrupamentos pelo total diário outrora calculado. Por meio desta última operação, obtém-se um valor percentual da recorrência.

O usuário ao configurar o sistema, opta por uma taxa de tendência, uma porcentagem que será comparada com o valor de recorrência que foi calculado anteriormente. Se o valor de recorrência individual de cada grupo for superior ao valor da taxa de tendência, significa que a execução desta tarefa no período determinado é superior ao requerido pelo usuário, identificando assim, uma tendência.

Esta tendência é registrada no banco de dados, o qual funciona como a memória da inteligência. Portanto o sistema é capaz de ir aprendendo com o tempo de forma autônoma, baseado nos dados que são captados e armazenados em sua memória.

A figura 3 representa uma das telas desenvolvidas no software. Indica a interface de análise dos padrões do usuário, onde é possível selecionar tanto semanalmente, quando diário, além da ação que se deseja analisar. É plotado então um gráfico, indicando qual dia e horário a ação têm-se como tendência, desta forma, a ação torna-se uma função a ser executada automaticamente.

Figura 3 - Tela de análise de tendências.

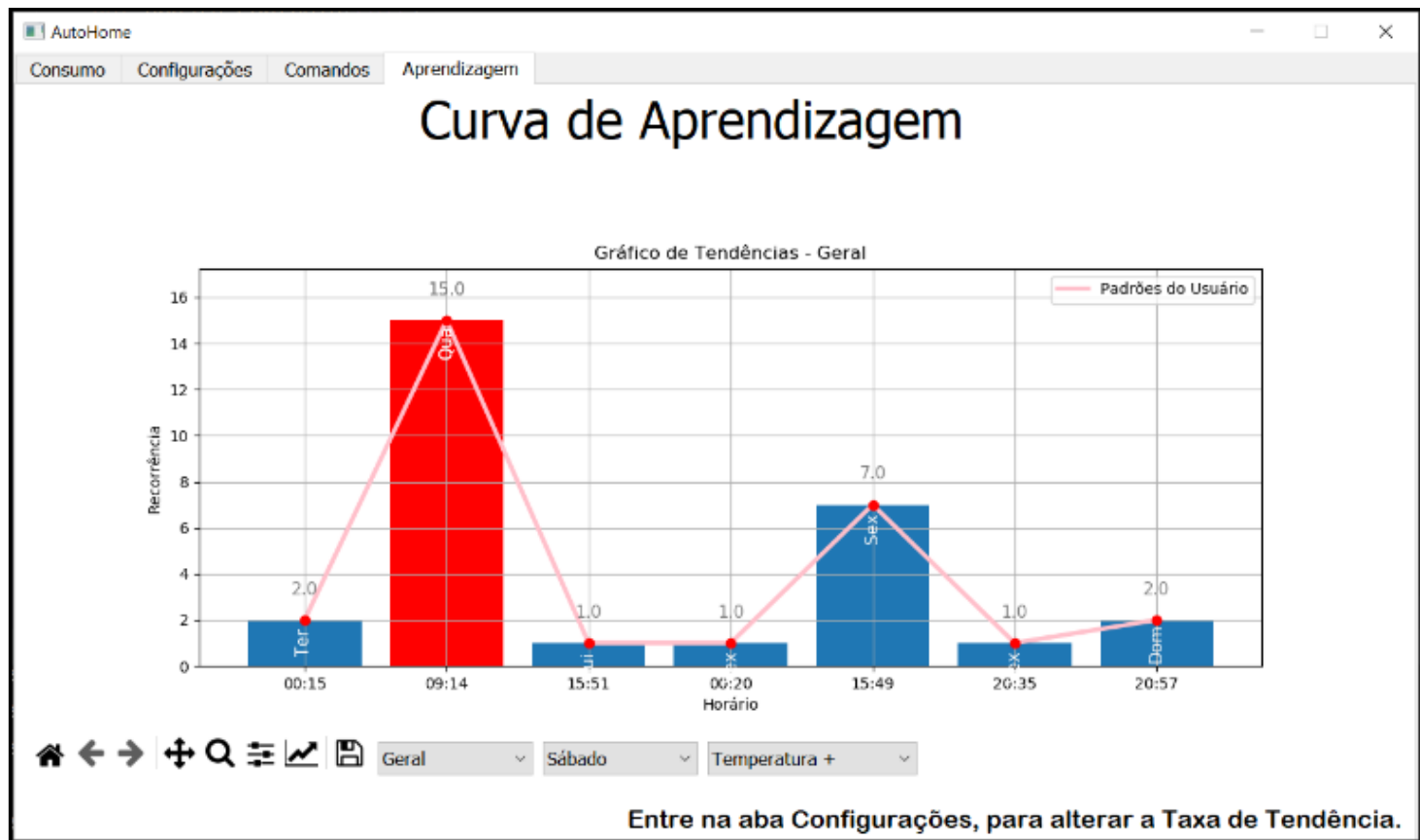

Fonte: Elaborado pelo autor. 


\section{CONCLUSÃO}

Devido ao avanço tecnológico, é possível desenvolver novas metodologias de aprendizado que permitem as máquinas e computadores abstrair informações e desenvolver lógicas, estruturar dados e obter conhecimento. Têm-se investido cada vez mais em áreas da inteligência artificial que permitam reproduzir estruturas do aprendizado humano.

Para reprodução de tal aprendizado, desenvolveu-se neste trabalho uma inteligência que, por meio de uma automação residencial, obtenha informações do padrão comportamental do usuário e de posse destes dados, aprenda a forma de agir do indivíduo para que possa reproduzir suas ações.

A inteligência desenvolvida utiliza uma técnica de machine learning conhecida como agrupamento, a qual resgata uma série da dados e estabelece filtragens, agrupamentos e análises baseados nestas informações. Esta corresponde à uma forma de aprendizagem semelhante à realizada pelos seres humanos. Estes observam um evento, suas consequências e a partir disto estabelecem padrões, paradigmas e conhecimento.

Os agrupamentos que são ordenados pela inteligência estabelecem tendências e permitem que a automação funcione por conta própria, seguindo os mesmos comportamentos do usuário que a utiliza. Desta forma, têm-se uma automação residencial autônoma que possui a capacidade de aprender, representando assim uma inovação na forma de aprendizagem.

\section{REFERÊNCIAS}

ALVARES, Luis Otávio; SICHMAN, Jaime S. Introdução aos sistemas Multiagentes. Anais.. Brasília: UnB, 1997.

LUGER, George F. Inteligência Artificial. 6. ed. São Paulo: Pearson, 2014. 631 p.

SILVA, Giovanni Cimolin da. Detecção e Contagem de Plantas utilizando técnicas de Inteligência Artificial e Machine Learning. 2017. 94 f. TCC (Graduação) - Curso de Engenharia Eletrônica, Departamento de Engenharia Elétrica e Eletrônica, Universidade Federal de Santa Catarina, Florianópolis, 2017. 


\title{
REPRODUCTION OF HUMAN LEARNING IN A RESIDENTIAL AUTOMATION THROUGH MACHINE
}

\begin{abstract}
The human individual is in constant learning. This being observes the environment in which it is inserted, identifies actions, analyzes its consequences, and in this way manages to abstract knowledge and define standards, this being its form of learning. In order to bring an innovation to the forms of learning, a lot has been invested in artificial intelligence in order to obtain computational methodologies that can represent this knowledge in a virtual way. One of these methods is known as Machine Learning, it allows to attribute to the machine, several ways to establish the learning. For example, there is the grouping, which refers to capturing information, analyzing it, grouping and identifying trends, similar to that of human beings in obtaining knowledge. One of the possible applications to this intelligence, corresponds to home automation. This work presents an autonomous intelligence that captures behavioral information from the user of the residence and establishes standards, so that it is possible to predict their actions and function automatically, thus establishing an intelligent and autonomous residence.
\end{abstract}

Keywords: Artificial intelligence. Machine Learning. Learning. Knowledge. Home automation. 